\title{
Les nouvelles dans la Vita de Benvenuto Cellini
}

La construction d'un roman personnel

\section{Cécile Terreaux-Scotto}

\section{(2) OpenEdition}

Journals

Édition électronique

URL : http://journals.openedition.org/cei/173

DOI : $10.4000 /$ cei. 173

ISSN : 2260-779X

Éditeur

UGA Éditions/Université Grenoble Alpes

\section{Édition imprimée}

Date de publication : 15 mars 2010

Pagination : 129-155

ISBN : 978-2-84310-164-9

ISSN : $1770-9571$

\section{Référence électronique}

Cécile Terreaux-Scotto, «Les nouvelles dans la Vita de Benvenuto Cellini », Cahiers d'études italiennes

[En ligne], 10 | 2010, mis en ligne le 15 septembre 2011, consulté le 27 mars 2021. URL : http:// journals.openedition.org/cei/173 ; DOI : https://doi.org/10.4000/cei.173 


\title{
LES NOUVELLES DANS LA VITA DE BENVENUTO CELLINI : LA CONSTRUCTION D'UN ROMAN PERSONNEL
}

\author{
Cécile Terreaux-Scotto \\ Université Stendhal - Grenoble 3
}

La matière de l'un et de l'autre est trop différente. Celle de la Nouvelle est un épisode, celle du roman une suite d'épisodes. Cet épisode que la Nouvelle se propose de peindre, elle le détache, elle l'isole. Ces épisodes dont la suite fait l'objet du roman, il les agglutine, il les relie. Il procède par développement, la Nouvelle par concentration [...] elle est un solo. Le roman est une symphonie.

Tels sont les termes que Paul Bourget emploie pour parler de la nouvelle et du roman ${ }^{\mathrm{I}}$. Envisager d'étudier la place et la fonction que rempliraient des nouvelles dans un roman apparaît dès lors bien paradoxal. Comment deux genres littéraires, qui sont certes tous deux narratifs, mais qui sont également aussi différents que le décrit Bourget pourraient-ils être réunis dans une même œuvre? Notre projet peut paraître aussi d'autant plus surprenant qu'il concerne un ouvrage généralement considéré comme une autobiographie dans les anthologies littéraires. Giulio Ferroni fait ainsi de la Vita de Benvenuto Cellini, orfevvre et sculpteur né à Florence en I500, la "première autobiographie moderne ${ }^{2}$ ».

De fait, la description que Cellini donne de la Vita dans son traité sur l'orfèvrerie ( ¡je me mis à écrire toute ma vie et mon origine et toutes les choses que j'avais faites en ce monde ${ }^{3} »$ ) correspond à la définition que Philippe Lejeune a établie de l'autobiographie, à savoir un «récit rétrospectif en prose qu'une personne réelle fait de sa propre existence,

I. D. Grojnowski, Lire la nouvelle, Paris, Nathan Université, 2000, p. II. Entre autres références critiques, citons : M. Bakhtine, Esthétique et théorie du roman, Paris, TEL Gallimard, 1978; P. Larivaille, «L'analyse morphologique du récit", Poétique, n I9, 1974, p. 368-388; P.-L. Rey, Le roman et la nouvelle, Paris, Hatier, 200I; T. Todorov et O. Ducrot, Dictionnaire encyclopédique des sciences du langage, Paris, Points Seuil, 1972.

2. G. Ferroni, Storia della letteratura italiana. Dal Cinquecento al Settecento, Turin, Einaudi, I99I, p. I64.

3. "io mi messi a scrivere tutta la vita mia e l'origine mio e tutte le cose che io avevo fatte al mondo" (B. Cellini, Dell'oreficeria, Opere, édité par B. Maier, Milan, Rizzoli, 1968, p. 717). 
lorsqu'elle met l'accent sur sa vie individuelle, en particulier sur l'histoire de sa personnalité ${ }^{4}$. Cellini lui-même insiste d'ailleurs à plusieurs reprises sur la nature autobiographique du texte qu'il dicte à un apprenti après avoir commencé à l'écrire de sa propre main. Les pronoms sujets et les adjectifs possessifs à la première personne en sont l'indice le plus évident : "J'écris ma vie tourmentée», "j'avais commencé à écrire ma Vie de ma main», «je lui dictais ma Vie», répète Cellini tout au long de la première page du manuscrit ${ }^{5}$. Cellini impose donc d'emblée à ses lecteurs une identité entre l'auteur (Benvenuto Cellini, artiste qui vécut principalement à Florence, Rome et Paris au XVI ${ }^{\mathrm{e}}$ siècle), le narrateur (Cellini qui raconte une histoire) et le personnage (Benvenuto que l'on voit agir), identité qui conditionne ce que Lejeune appelle «le pacte autobiographique» (Lejeune, p. I5-19 et p. 26-27).

Mais une lecture, même rapide, de la Vita permet d'y voir à l'œuvre des genres littéraires très variés. Du roman antique ou chevaleresque à la comédie, de la tragédie à l'hagiographie, en passant par la poésie et la nouvelle, Benvenuto Cellini fait du récit de sa vie une véritable mosaïque littéraire ${ }^{6}$. Nous nous proposons d'examiner ici comment l'exploitation d'un genre littéraire particulièrement exploré au Xvi ${ }^{\mathrm{e}}$ siècle - la nouvelle héritée de Boccace - parcourt la trame narrative de la Vita. Si l'infiltration de ce genre littéraire fragilise en apparence la nature autobiographique du texte, l'analyse de la signification de chacune des nouvelles étudiées ainsi que de leur fonction dans l'économie générale de l'œuvre permettra cependant de donner un sens général au projet de l'artiste.

Certains passages de la Vita apparaissent comme de courtes excroissances narratives brisant en apparence la continuité narrative des épisodes qui les précèdent et qui les suivent.

C'est le cas, par exemple, du chapitre I-9. À la fin du chapitre I-87, Cellini évoque l'activité d'orfèvre du personnage Benvenuto qui est alors logé chez Francesco Castoro à Sienne : «je m’en allai chez cet homme de

4. Ph. Lejeune, Le pacte autobiographique, Paris, Seuil, 1975, p. I4.

5. "Questa mia vita travagliata io scrivo", "Io avevo cominciato a scrivere di mia mano questa mia Vita", "gli dittavo la Vita mia» (B. Cellini, Vita, édité par E. Camesasca, Milan, BUR, 2007, p. 79 et p. 80). Lorsque nous ferons désormais référence à cette édition dans le corps du texte, seul le numéro de page figurera entre parenthèses.

6. Voir à ce sujet A. Cicchetti, «La Vita di Benvenuto di Mº Giovanni Cellini fiorentino scritta (per lui medesimo) in Firenze", dans Letteratura italiana. Le opere, édité par A. Asor Rosa, vol. II, Dal Cinquecento al Settecento, Turin, Einaudi, 1993, p. 54I-562, en particulier p. 545-549 et p. 553-554.

7. Par commodité, nous suivons la tradition éditoriale qui a réparti la matière en chapitres, même s'ils ne correspondent à aucune indication dans le manuscrit de Cellini. 
bien et je restai avec lui plusieurs jours [...] toujours en travaillant l'art de l'orfèvrerie ${ }^{8} »$ (p. 96). Au début du chapitre I-Io, le narrateur reprend les mêmes termes pour réinscrire les deux extraits dans une parfaite unité narrative et thématique, soulignée par l'emploi du gérondif et la reprise en écho de l'adverbe "pure": «je continuais à me consacrer à l'art de l'orfèvrerie 9 " (p. 99). Cellini procède de façon semblable lorsqu'il parle de son frère. Tout comme il écrit à la fin du chapitre I-8 que « $[s]$ on frère avait des notions de latin ${ }^{10}$ "(p. 96), il répète à son sujet au début du chapitre I-Io que " $[s]$ on frère Cecchino, comme [il l'a] dit ci-dessus, avait reçu des notions de latin ${ }^{\mathrm{II}} "$ (p. 99). L'irruption du discours dans l'histoire ${ }^{12}$, par le biais du narrateur qui rappelle qu'il a déjà abordé cette question ("comme je l'ai dit ci-dessus»), sert à souligner le lien entre les deux chapitres. Dans ce contexte, le chapitre I-9 apparaît comme une pure digression, d'autant qu'il est introduit par la même formulation que celle qui ouvre le chapitre I-8 : «en ce temps-là ( «in questo tempo», p. 94 et p. 96). L'imprécision de l'indication chronologique permet à Cellini d'insérer artificiellement le chapitre I-9, dont l'absence ne nuirait en rien à l'histoire racontée dans la Vita ${ }^{13}$.

Il en va de même pour les chapitres I-27 à I-30 qui animent la période consacrée à la peste ayant sévi à Rome en 1523 . À la fin du chapitre I-26, Cellini évoque l'activité artistique polymorphe du personnage dont il raconte la vie : Benvenuto apprend à travailler les sceaux auprès de Lautizio, les médailles grâce à Caradosso, l'émail avec Amerigo Righi. Voici les derniers mots du chapitre I-26: «moi, je m’ingéniais de toutes mes forces à exercer de façon égale toutes ces professions; et le moment venu je montrerai ce que j'ai fait, de la façon dont je le dis ${ }^{14}{ }_{\text {}}$ (p. 139). Cette phrase, qui résume l'affairement de l'artiste, annonce en réalité le début du chapitre I-3I : «moi je me consacrais avec le plus grand soin et la plus grande

\footnotetext{
8. «me n'andai da questo uomo dabbene e stetti seco certi giorni [...] pure lavorando dell'arte dell'orefice».

9. "attendendo pure all'arte de l'orefice».

Io. «il mio fratello aveva principio di lettere latine».

II. "mio fratello chiamato Cecchino, come di sopra dissi, avendogli fatto dare principio di lettere latine».

I2. Nous reprenons ici la distinction proposée par Benveniste, l'histoire étant l'ensemble des événements racontés, par rapport au discours qui met en rapport un narrateur, chargé de relater l'histoire, et un lecteur, qui en prend connaissance.

13. Nous adoptons ici la terminologie exposée par Genette. "L'histoire» désigne "le contenu narratif», ce qui est raconté; le «récit» indique «le signifiant, l'énoncé, le discours ou le texte narratif lui-même». Enfin la «narration" est «l'acte narratif lui-même, l'ensemble de la situation réelle ou fictive dans laquelle il prend place». (G. Genette, Figures III, Paris, Seuil, I972, p. 72.)

I4. «io, ingegniatomi con tutto il mio potere di tutte queste professione equalmente operare; e al suo luogo mostrerrò tal cosa aver fatta, sì come io dico ".
} 
attention à me former à ces arts divers et variés dont j'ai parlé plus haut ${ }^{15}$ " (p. I52-I53). La continuité entre les chapitres I-26 et I-30 est là encore renforcée par l'apparition brutale du narrateur dans le récit, d'abord au moyen d'une anticipation («le moment venu je montrerai») puis par un rappel («dont j'ai parlé plus haut») ${ }^{16}$.

Entre ces deux notations, Cellini développe quatre scènes qui scandent l'évolution de l'épidémie. Aux premiers signes de la peste («en ce temps-là la maladie pestilentielle revint ${ }^{17} », p$. 139), Benvenuto part se distraire à la chasse aux pigeons. Au moment où l'épidémie se développe («la peste avait commencé à Rome ${ }^{\mathrm{I}}$ ", p. I42), il rencontre le charlatan Iacomo da Carpi. Alors que la peste s'installe dans la durée («la peste continua ensuite pendant de nombreux mois ${ }^{19}$ », p. I44), il contracte une maladie vénérienne avec la servante de Faustina. Mais la fin de l'épidémie suscite un regain de vitalité chez les habitants de Rome : «la peste avait pratiquement déjà cessé, de sorte que ceux qui se retrouvaient vivants se caressaient très joyeusement ${ }^{20}$ » (p. I47). Cellini illustre ces changements à travers le récit d'un souper entre artistes, sur lequel nous reviendrons. Or, si ces quatre épisodes semblent suivre le fil rouge constitué par la peste, ils fonctionnent en réalité de façon indépendante les uns des autres.

Autonomes par rapport à l'ensemble de la Vita, ces digressions narratives, plus ou moins longues, sont aussi auto-suffisantes, au sens où elles racontent en quelques pages ou quelques lignes un récit complet, à l'instar de la nouvelle, cette «forme simple, close sur elle-même, [qui] délimite un espace de fiction autarcique", qui se concentre sur les événements en un espace beaucoup plus restreint que celui du roman, et dans laquelle "chaque histoire vaut pour elle-même et se suffit à elle-même" (Grojnowski, p. 56).

Mais il est intéressant de constater que ces digressions narratives s'insèrent de manière plus ou moins plane dans la trame narrative générale de la Vita.

Dans le chapitre I-IO5, par exemple, Cellini raconte comment un religieux, frère Pallavicini, cherche à convaincre Benvenuto de ne pas respecter le pacte qu'il a conclu avec son geôlier et de s'enfuir. Or, cet épisode

I5. «io attendevo con ogni sollecitudine e diligenzia a farmi pratico in quella diversità e differenzia di arte, che di sopra ho parlato".

I6. Sur les figures techniques de la prolepse et de l'analepse, voir Genette, op. cit., p. 77-I20.

17. «in questo tempo si risentì un morbo pestilenziale».

18. "era cominciato la peste in Roma».

19. "Seguitando apresso la peste molti mesi».

20. "di già era quasi cessata la peste, di modo che quelli che si ritrovavono vivi molto allegramente l'un l'altro si carezzavano». 
ne dévie pas le récit de sa trajectoire spatio-temporelle dans la mesure où il est situé au moment où Benvenuto est emprisonné, pour la première fois, au château Saint-Ange. Il constitue cependant une digression et une péripétie ajoutée au niveau de l'action dans la mesure où les rapports qui s'instaurent entre les personnages menacent de rompre l'équilibre auquel le prisonnier était parvenu (évoluer librement dans l'enceinte du château sans chercher à s'évader conformément à l'accord passé avec le châtelain). Aussi Cellini présente-t-il d'emblée l'enjeu qui oppose les deux personnages de ce récit qui se déroule comme une petite nouvelle à part entière : Benvenuto va-t-il continuer à résister aux pressions du religieux et pourrat-il rester fidèle à la parole qu'il a donnée? (p. 346). Dans un deuxième temps, Cellini orchestre les péripéties qui vont transformer la situation initiale. Pour ce faire, il introduit un élément nouveau : voyant que la simple suggestion de l'évasion ne suffit pas, le religieux tente d'aliéner la conscience de Benvenuto en le charmant par la lecture des sermons de Savonarole. Cette phase de déséquilibre se poursuit au moment où frère Pallavicini touche le point sensible de Benvenuto en doutant de ses compétences techniques et en le mettant au défi de pouvoir forcer la serrure. Les conséquences de cette action introduisent alors une dynamique de rupture : par orgueil, Benvenuto ne pense plus à respecter la parole qu'il a donnée à son geôlier mais à prouver à son co-détenu qu’il a le talent nécessaire pour s'enfuir : "je lui montrai avec facilité tout ce que j'avais dit ${ }^{2 \mathrm{I}}$ " (p. 347). Ces péripéties se terminent sur les moyens utilisés par les personnages pour tenter de réparer ce qui a été bouleversé lorsque le forgeron a découvert les clés fabriquées par Benvenuto : d'un côté, le maître des lieux enferme l'artiste sous les injures des gardiens, mais d'un autre côté un serviteur témoigne en sa faveur. L'action combinée de ces personnages, qui tiennent les rôles complémentaires d'opposant et d'adjuvant, permet un retour à la situation initiale comme le souligne explicitement Cellini : «le dit Châtelain [...] me donna la permission d'être dans les mêmes conditions que celles où j'étais auparavant ${ }^{22}$ » (p. 349). Sauf que dans l'intervalle constitué par la digression, Benvenuto a pu faire montre de son talent de sculpteur de clés.

Si ce chapitre I-Ios s'inscrivait dans une continuité spatio-temporelle avec les épisodes précédents et suivants (le château Saint-Ange en 1537), les chapitres I-63 à I-70 ont au contraire leur propre unité narrative, parfaitement reconnaissable, à l'intérieur de la structure globale de la Vita.

2I. "gli mostrai con facilità tutto quello che io avevo detto".

22. «il detto Castellano [...] me allargò inel medesimo modo che io mi stavo in prima". 
Centrés autour d'un seul événement, la passion amoureuse de l'artiste, ces chapitres constituent une forte digression thématique par rapport au fil du récit. Avant de raconter la rencontre de Benvenuto avec Angelica (introduite, de façon symptomatique, par la notation chronologique habituelle aussi vague qu'artificielle "in questo tempo»), Cellini fait allusion à la médaille que Benvenuto fabrique pour le pape Clément VII : "Je m'étais mis à faire son portrait, et je lui faisais une médaille en secret ${ }^{23} »$ (p. 239). Or, significativement, après avoir raconté la rupture entre les amants, Cellini remet Benvenuto au travail, et plus précisément à la fabrication de cette même médaille: "Je m'en allai chez moi, me mettant à finir la médaille de la tête du pape Clément que j'avais déjà commencée ${ }^{24}$ (p. 256). Tout se passe alors comme si la relation entre Benvenuto et Angelica n’avait été qu'une parenthèse purement accessoire, dont le récit aurait très bien pu faire abstraction puisqu'il se termine comme il avait commencé, à savoir par une allusion à la position d'artiste-courtisan du héros de la Vita.

Le chapitre I-9 quant à lui, est marqué par une construction temporelle très complexe qui souligne le statut particulier de cette digression. Ce chapitre commence par un déplacement de Benvenuto à Bologne, qui revient ensuite à Florence "au bout de six mois" ("in capo di sei mesi», p. 97) après avoir appris la musique. La transformation qui survient bouleverse d'autant plus la situation initiale qu'elle est due au hasard. C'est en effet «un jour parmi les autres» («un giorno infra li altri», p. 97) qu'un joueur de fifre nommé Pierino défie le père de Benvenuto en proclamant que celui-ci est davantage doué pour l'orfèvrerie que pour la musique. Un affrontement verbal oppose alors les deux protagonistes, qui redirigent systématiquement l'attaque qu'ils reçoivent sur celui qui l'a proférée en premier, sur le modèle des répliques échangées du tac au tac dans les dialogues qui animent traditionnellement les nouvelles dans la lignée du Décaméron: de la même façon que Pierino traite Giovanni de fou, Giovanni à son tour taxe les enfants de Pierino de folie; alors que Pierino prévoit que ses enfants aideront les enfants de Giovanni, Giovanni rétorque que ce sont les enfants de Pierino qui se traîneront devant ses propres enfants parce qu'il prédit un avenir fort sombre pour leur père. Une série d'indications chronologiques introduit enfin progressivement dans le récit la réalisation de la prophétie de Giovanni Cellini. "Alors que le mois n’était pas terminé» («Non arrivato il mese intero», p. 98),

23. «Io m'ero messo a ritrarlo, e gli facevo una medaglia segretamente».

24. "Io me ne andai a casa mia, mettendomi a finire la medaglia, che di già avevo cominciata, della testa di papa Clemente». 
Pierino a un accident dont il meurt "peu de temps après» ("poco tempo appresso ", p. 99). Surtout, une projection très nette dans le futur dévie le récit de sa trajectoire temporelle puisque c'est "plusieurs années plus tard» («alquanti anni a presso », p. 99), lorsque le fils de Pierino se traîne aux genoux de Benvenuto Cellini, que la prophétie de Giovanni se réalise pleinement. Il faut d'ailleurs le retour de Benvenuto sur le passé par le souvenir ${ }^{25}$ pour mettre un terme aux péripéties et assurer à la fois la fin de l'épisode et le retour à la ligne temporelle de l'histoire initiale : à ce moment-là Cellini peut passer directement, après un bref commentaire, au chapitre I-IO.

Cet exemple montre que même s'il y a parfois une projection dans le futur, seule la durée de l'action racontée délimite le temps de la digression, comme celui d'une nouvelle. Une fois l'action achevée, la digressionnouvelle ne peut que prendre fin puisqu'elle se suffit à elle-même.

Il en va de même lorsque la nouvelle raconte une action qui se déroule sur une durée très réduite. L'histoire du chapitre I-30 par exemple est concentrée sur quelques heures d'une soirée dominicale. De la même façon, l'épisode où Benvenuto, alors âgé de trois ans, s'empare d'un scorpion, se déroule pendant un laps de temps extrêmement court, de l'ordre des minutes. Et pourtant l'épisode est construit comme une nouvelle. En premier lieu une situation initiale, au cours de laquelle Benvenuto, alors âgé de trois ans, aperçoit un scorpion sorti d'une canalisation. En second lieu une série de péripéties qui s’enchaînent rapidement : Benvenuto saisit l'animal et le montre à son grand-père. La tension est là à son comble puisque le vieillard, raconte Cellini, "à cause de sa grande frayeur et de sa tendresse pour moi manqua tomber raide $\operatorname{mort}^{26}{ }$, cet adjectif constituant l'acmé du texte (p. 88). Le lecteur entre là dans le deuxième moment des péripéties, où le grand-père cherche à convaincre Benvenuto de lui donner l'animal. L'intervention inattendue du père de l'enfant, qui coupe les pinces et la queue du scorpion, constitue le tournant décisif qui ramène à la situation initiale, assorti du commentaire qui conclut généralement les nouvelles. Sauf que là encore, dans l'intervalle, la digression a pu mettre l'accent sur l'annonce d'un avenir prometteur pour Benvenuto - nous y reviendrons.

25. "et ensuite avec des larmes je me souvins de l'heureuse situation qu'avait Pierino lorsque mon père lui avait dit ces mots, à savoir que les enfants de ce Pierino iraient demander de l'aide à ses enfants vertueux" ("e appresso con lacrime mi ricordai il felice istato che Pierino aveva, quando mio padre li disse tal parole, cioè che i figliuoli del ditto Pierino ancora andrebbono per la mercé ai figliuoli virtuosi sua»), p. 99.

26. "per il grande spavento e per la gelosia di me, fu per cader morto". 
Au terme de l'analyse d'un aspect singulier de la structure de la Vita, plusieurs passages apparaissent ainsi comme de pures parenthèses narratives. Leur absence ne nuirait en effet aucunement ni à la compréhension de la trame narrative ni à la succession logique des événements, pas plus que leur présence n'apporte des éléments susceptibles de modifier le déroulement des actions qui les précèdent ou qui les suivent. Ces digressions sont des "catalyses" au sens que donne Roland Barthes à ce terme, c'est-à-dire que ce sont des éléments qui remplissent l'espace narratif entre deux "fonctions cardinales", lesquelles constituent la trame même du récit dans la mesure où «l'action à laquelle elle[s] se réfère[nt] ouvre[nt] (ou maintienne[nt] ou ferme[nt]) une alternative conséquente pour la suite de l'histoire ${ }^{27} »$. Nous avons pu aussi déterminer que leurs caractéristiques narratives en faisaient des sortes de nouvelles en miniature. Quant à l'étude des modalités de leur inscription dans la trame narrative d'ensemble de la Vita, pour variées qu'elles soient, elle a montré que "chaque fois le lecteur fait l'expérience d'une temporalité différente mais toujours unifiée. Comme le temps du théâtre ou du cinéma de fiction, le temps de la nouvelle se définit par son unité dramatique» (Grojnowski, p. 88).

Pourquoi, dès lors, Cellini parsème-t-il son autobiographie de textes qui ne sont a priori ni nécessaires ni suffisants à l'économie de la Vita?

Cette question est d'autant plus pertinente que ces incursions vers un autre genre littéraire - celui de la nouvelle, en l'occurrence - ne peuvent que fragiliser en apparence la nature de l'œuvre que Benvenuto Cellini a voulu autobiographique.

Rappelons que pour que le "pacte autobiographique» que l'auteur passe avec ses lecteurs soit respecté, il faut que subsiste, toujours, l'identité entre l'auteur Benvenuto Cellini, personne ayant réellement existé à la Renaissance, le narrateur Cellini, instance qui dirige le récit, et le personnage Benvenuto, qui accomplit ou subit l'action.

Cette identité est respectée par exemple dans la digression constituée par le chapitre I-30, où la participation de Benvenuto à une scène collective (le souper entre artistes), exprimée par la fréquence des pronoms personnels à la première personne du pluriel, fait de lui un personnage qui raconte, rétrospectivement, ce qu'il a vécu ce soir-là. De plus, la mention de plusieurs noms appartenant au milieu artistique indique que Cellini est le témoin privilégié de la dolce vita menée à Rome par les artistes, au

27. R. Barthes, «Introduction à l'analyse structurale des récits», dans L'analyse structurale du récit, Communications 8, Paris, Points Seuil, 1966, p. 15. 
point qu'Angela Biancofiore peut écrire que la Vita est une "autobiographie ayant valeur historique ${ }^{28}{ }$. Cet aspect confirme dès lors au lecteur, de façon implicite, l'identité entre le narrateur et l'auteur.

L'identité auteur-narrateur-personnage est tout autant respectée dans le chapitre I-9, à la construction temporelle pourtant singulière. Benvenuto y reste à la fois le narrateur-témoin de la scène qui a opposé son père à Pierino d'une part, le personnage-enjeu de leur conflit et le protagoniste héroïque d'une action qui le met en valeur lorsqu'il donne l'aumône au fils de Pierino d'autre part, l'auteur-narrateur enfin, qui met en garde les lecteurs en soulignant l'importance de croire aux prédictions des hommes de bien, sous peine de s'attirer des ennuis. C'est que l'une des fonctions traditionnelles de la nouvelle est précisément d'édifier le lecteur ${ }^{29}$.

Ailleurs cependant, il arrive que le sujet de l'énonciation ne soit pas le même que le sujet de l'énoncé. Il semble alors qu'il y ait là une véritable entorse au pacte autobiographique. Emblématiques de cette dislocation de l'identité auteur-narrateur-personnage sont les chapitres I-II2 et I-II3, qui contiennent deux récits parallèles sur le même thème (Benvenuto a décapité un pigeon en un seul tir) mais racontés tour à tour depuis un point de vue différent, d'abord par Pier Luigi Farnese puis par Benvenuto Cellini, le premier affirmant que Benvenuto visait en réalité un cardinal, le second cherchant à apporter un démenti formel à cette version ${ }^{30}$.

Après s'être évadé du château Saint-Ange, Benvenuto a trouvé refuge auprès du cardinal Cornaro. Le gouverneur de Rome lui rend visite puis s'en va, seul, plaider la cause de l'artiste auprès du pape. Mais le fils de ce dernier, Pier Luigi Farnese, prononce un réquisitoire implacable contre Benvenuto : le protagoniste de la Vita est présenté comme un être démoniaque assoiffé de sang qui, pour masquer l'assassinat prémédité d'un cardinal, avait tiré sur un pigeon ${ }^{31}$.

28. A. Biancofiore, Benvenuto Cellini artiste-écrivain : l'homme à l'œuvre, Paris, L'Harmattan, I998, p. 38.

29. Comme le suggère l'introduction au Décaméron, dans laquelle Boccace fait des nouvelles l'équivalent, entre autres, des "paraboles». Voir sur ce point L. Battaglia Ricci, "'Una novella per esempio'. Novellistica, omiletica e trattatistica nel primo Trecento", dans Favole parabole istorie. Le forme della scrittura novellistica dal Medioevo al Rinascimento, Atti del Convegno di Pisa (26-28 octobre 1998), Rome, Salerno editrice, 2000, p. 49 .

30. Pour une analyse de ces épisodes, voir A. Godard, "Sur quelques passages de la Vita de Cellini. Du danger du développement romanesque dans l'autobiographie», Filigrana, L'écrit et la mémoire, nº 4, 1997, p. 60-66.

3I. «Et comme le Cardinal fut prévenu, il se leva tout de suite. Benvenuto, pour ne pas laisser paraître ses projets, tira sur un pigeon sauvage qui couvait dans une niche en haut du palais, et il toucha ce pigeon à la tête : chose impossible à croire.» («E perché il Cardinale ne fu avvertito, si levò subito. Benvenuto, perché e’ non si paressi tal cosa, tirò a un colombo terraiuolo che covava in una buca sù alto del palazzo, e dette al ditto colombo in nel capo: cosa inpossibile da poterlo credere", p. 367-368.) 
Au niveau de la narration, le texte procède par enchâssement ${ }^{32}$, c'està-dire que ce que raconte Pier Luigi Farnese à propos du personnage Benvenuto est inclus dans l'histoire que le narrateur Cellini raconte depuis le début de la Vita. Rapporté au style direct introduit par des guillemets et une formule déictique ( Le seigneur Pierluigi ajouta alors ces mots : "Bienheureux Père ${ }^{33} .$. " ", p. 367), le discours du personnage Farnese se substitue au discours du narrateur Cellini qui n'est présent qu'à travers les guillemets qu'il referme lorsque le fils du pape cesse de parler. Écarté pour un temps, le narrateur Cellini est également provisoirement séparé du personnage Benvenuto.

Tout d'abord, dans l'économie interne de l'histoire, aucun indice n'explique comment Benvenuto a eu connaissance des mots prononcés par Pier Luigi Farnese, alors qu'il est censé, en tant que personnage-narrateur, avoir été témoin de la scène. Mais il a été clairement indiqué que le gouverneur qui va entendre Pier Luigi Farnese a laissé seul le personnage Benvenuto : «il me quitta», rapporte ce dernier ("partitosi da me», p. 367). À ce moment-là, la convergence entre l'histoire (ce qui est raconté dans la $V i t a$ ) et la narration (l'acte de raconter) n'est donc plus possible. Un auteur omniscient, auquel rien des lieux, des événements et des personnages qui peuplent la Vita n'échappe, se substitue dès lors au personnage-narrateur, au point, d'ailleurs, qu'il peut décrire l'état d'esprit du pape. Pourtant, rappelons-le, le personnage Benvenuto n'était pas présent au moment où Farnese a parlé. Tout se passe donc comme si le narrateur était dissocié du personnage, ce qui constitue une entorse au "pacte autobiographique».

Mais ce n'est pas tout. Car dans le cadre de ces événements racontés dans un récit second par rapport au récit premier avec un narrateur autre que le narrateur principal ${ }^{34}$, il ne peut être question de Benvenuto qu'à la troisième personne du singulier. C'est d'ailleurs selon cette modalité qu'est lancé le réquisitoire de Farnese contre Benvenuto :

[...] si vous le libérez, il vous en fera de plus belles, parce que c'est un type d'homme beaucoup trop audacieux. Je vais vous raconter une autre histoire que vous ne connaissez pas. Votre Benvenuto, avant d'être en prison, avait un différend avec un gentilhomme du cardinal Santa Fiora [...] $]^{35}$. (p. 367)

32. Opposé à l'enchâssement, qui fonctionne sur un rapport syntaxique de subordination, se trouve l'enchâ̂nement, qui consiste à juxtaposer différentes histoires coordonnées entre elles (T. Todorov, "Les catégories du récit littéraire", dans L'analyse structurale du récit, Communications 8, Paris, Points Seuil, I966, p. I25-I51, ici p. I46).

33. "Il signior Pierluigi allora aggiunse dicendo: "Beatissimo Padre...”"

34. Événements que Genette appelerait «métadiégétiques» (op. cit., p. 238-239).

35. «[...] se voi lo liberate, egli ve ne farà delle maggiori, perché questo è uno animo d'uomo troppo aldacissimo. Io ve ne voglio contare un'altra, che voi non sapete. Avendo parole questo vostro Benvenuto, innanzi che 
L'effacement du «je» de Benvenuto est symptomatique de la volonté de Cellini de se désolidariser du personnage que décrit Farnese. Le style indirect aurait permis au narrateur Cellini de continuer à rester aussi le personnage Benvenuto par l'emploi de la première personne du singulier, dans des phrases du type : "Farnese dit que si le pape me faisait libérer, je lui en ferais de plus belles parce que je suis un type d'homme [...].» Mais le style direct introduit une mise à distance, comme si l'auteur de la Vita ne se reconnaissait pas dans le portrait - négatif et dévalorisant que brosse de lui Farnese. Car tel est probablement le message qu'il veut adresser, implicitement, à ses lecteurs.

Cette hypothèse est confirmée par la réapparition aussi brutale que rapide du pronom personnel «je» qui incarne dans un deuxième temps un Benvenuto voulant rétablir ce qu’il présente comme la seule et unique vérité : "Je ne veux pas manquer de faire valoir mon bon droit avec justesse et honnêteté ${ }^{36}$ " (p. 368). Mais ce «je» est moins celui d'un personnagenarrateur que celui d'un auteur tout puissant. Car l'irruption du présent de l'indicatif ("je veux, voglio») dans un texte jusque-là au passé, et qui s'apprête à continuer au passé, exprime l'irruption du discours, de l'instance narrative, dans le récit. Comme s'il y avait là une sorte de débordement incontrôlable du Cellini-auteur devant l'injustice qui était faite à Benvenuto dans le portrait dévalorisant qui venait d'être fait de lui.

De plus, la mise en relief de la narration, dont les ressorts apparaissent ici de façon explicite, marque la présence de l'auteur Benvenuto Cellini tel qu'il est au moment où il raconte la Vita. Conscient du tort que peuvent lui causer les accusations qu'il a lui-même fait formuler par Pier Luigi Farnese, l'artiste annonce au lecteur qu'il va y répondre. Renouant avec le pacte autobiographique, il reprend pour un temps sa place de narrateur, tout en écartant de la scène narrative celui qu'il considère comme un calomniateur. S'ouvre alors, dans le chapitre I-II3, une digression semblable à celles que nous avons définies dans la première partie de cette étude.

Tout d'abord, il s'agit d'une excroissance par rapport au récit puisque l'épisode est encadré, au début et à la fin, par l'image du pape qui réagit aux propos de son fils. Voici en effet la phrase qui précède la réapparition de Cellini narrateur dans le texte : "Le pape était empli de colère et ne disait pas un $\operatorname{mot}^{37} »$ (p. 368). On peut ensuite lire l'anecdote du pigeon,

lui fussi prigione, con un gentiluomo del cardinal Santa Fiore [...].»

36. "Io non voglio mancare che io non dica le mie ragione giustamente e santamente».

37. «il Papa stava gonfiato e non parlava nulla». 
racontée, cette fois-ci, du point de vue de Benvenuto. Le récit initial redémarre sur la reprise, presque à l'identique, des termes concernant le pape, comme si le témoignage de Benvenuto n'avait pas eu lieu : «Le pape empli de colère et assombri, pensait à ce que lui avait dit son fils ${ }^{38}$ " (p. 369).

Par ailleurs, en quelques lignes, Cellini décrit une situation initiale (l'artiste refuse d'exécuter un travail pour l'ami du cardinal Santa Fiora) puis des péripéties qui se déroulent deux jours plus tard (Benvenuto raconte comment il a réussi à atteindre, contrairement à son rival l'orfevre Giovan Francesco della Tacca, la cible que constituait le pigeon). Ces données constituent implicitement les arguments de l'auto-plaidoyer et de la réhabilitation de Benvenuto. Il apparaît en effet que le client insatisfait de Benvenuto a transformé, pour se venger, les circonstances de la mort du pigeon. Le lecteur est alors invité à bénéficier d'une sentence moralisatrice et universelle (pour laquelle Benvenuto a servi d'exemple) sur le modèle des leçons que présentent bien souvent les nouvelles (Grojnowski, p. 156): «De sorte que le monde peut voir quelles différentes voies la fortune prend lorsqu'elle veut entreprendre de tourmenter un homme ${ }^{39}$ " (p. 369).

Sur la ligne d'un récit racontant la colère du pape contre Benvenuto, qui est momentanément absent en tant que personnage actif de l'histoire, vient donc se greffer un second micro-récit, autonome et auto-suffisant. Le lecteur voit de nouveau le personnage Benvenuto agir. De plus, le rôle de narrateur qui avait été tenu précédemment par Pier Luigi Farnese revient à Benvenuto. On passe par conséquent d'un narrateur au second degré qui raconte une histoire d'où il est absent à un narrateur toujours au second degré, mais qui raconte sa propre histoire ${ }^{40}$. Si bien qu'en prenant la place de Farnese, Benvenuto devient finalement un personnage qui raconte, un personnage qui se fait narrateur pour raconter une nouvelle. Il n'est donc pas seulement le héros de l'histoire qu'il raconte, mais aussi son metteur en scène. Par un effet de miroir, la nouvelle racontée par le personnage Benvenuto constitue alors comme une véritable mise en abîme de l'autobiographie racontée par le narrateur Cellini qui dirige le récit de l'ensemble de la Vita.

D'ailleurs, dans la Vita les capacités oratoires de Benvenuto suscitent souvent l'admiration de son entourage. C'est ainsi que François I ${ }^{\text {er }}$ le gratifie du vocatif affectueux «mon ami» (p. 458) après qu'il l'a entendu lui

38. «il Papa gonfiato e ingrogniato, stava considerando quel che gli aveva detto il suo figliuolo».

39. "Sì che vegga il mondo, quando la fortuna vuol torre assassinare uno uomo, quante diverse vie la piglia».

40. Pour Genette, Farnese serait un narrateur intradiégétique-hétérodiégétique, alors que Cellini serait ici un narrateur intradiégétique-homodiégétique. 
décrire une partie de la Nymphe de Fontainebleau. En cela il comble les aspirations de Benvenuto, qui «ayant plu par l'action voulait bien plaire autant par la parole ${ }^{4 \mathrm{I}}$ ( (p. 459). De même, c'est pour ses qualités d'orateur que Benvenuto se voit promettre cinq cents écus d'or par l'empereur Charles Quint. Il a su en effet parler «de différentes choses, toutes vertueuses et plaisantes ${ }^{42}$ » (p. 3ro) alors que Durante Duranti avait déclenché l'hilarité du souverain parce qu'il s'était exprimé «d'une façon si disgracieuse et avec certains mots de Brescia, sa langue s'entortillant dans sa bouche, de telle sorte qu'on ne vit ni n'entendit jamais rien de pire ${ }^{43}$ " (p. 309).

L'exploitation dans la Vita du genre littéraire de la nouvelle entraîne donc parfois un effritement du pacte autobiographique. Nous avons vu, en particulier, qu'il peut être question de notre auteur-narrateur-personnage à la troisième personne du singulier, alors que la caractéristique la plus évidente de l'autobiographie est au contraire justement l'emploi de la première personne. Dans cette perspective, un épisode de la Vita est toutefois encore plus surprenant: le chapitre I-3, dans lequel Cellini emploie luimême la troisième personne grammaticale pour parler de Benvenuto. Ce passage est d'autant plus intéressant qu'il ne s'agit rien de moins que de la naissance de notre artiste.

Dans le cadre de l'autobiographie, la venue au monde du personnagenarrateur, tout comme sa mort, est de l'ordre de l'indicible. La première, parce qu'il n'existait encore pas, la seconde, parce qu'il n'existe plus. Pour le récit de sa naissance ${ }^{44}$, le narrateur peut toutefois s'appuyer sur des informations qui lui ont été fournies par des tiers. C'est d'ailleurs ainsi que procède Cellini lorsqu'il raconte la scène où Benvenuto enfant s'est emparé d'un scorpion. L'emploi d'une troisième personne du pluriel indistincte souligne que le narrateur restitue des éléments qu'on lui a rapportés, parce que sa mémoire ne pouvait pas lui permettre d'en avoir connaissance : "Ils disent que je courus fort joyeusement vers mon grand-père en disant : "Regarde, grand-père chéri, mon beau petit crabe!" 45 » (p. 88).

Mais aucune notation ne figure en ce sens lorsqu'est racontée la naissance de Benvenuto. De plus, en dehors de l'adjectif possessif à la première

\footnotetext{
4I. "essendo piaciuto col fare volev[a] bene altretanto piacessi il [suo] dire».

42. "di diverse cose tutte virtuose e piacevole".

43. "con tanto isgraziato modo e con certe sue parole bresciane, annodandosigli la lingua in bocca, che mai si vidde e sentì peggio ".

44. Sur cette catégorie de récit, voir le chapitre qu’y consacre Lejeune dans Moi aussi, Paris, Seuil, 1986.

45. "Dicono che con gran festa io corsi al mio avo, dicendo: "Vedi, nonno mio, il mio bel granchiolino!" " (C'est nous qui soulignons.)
} 
personne du singulier qui figure devant le mot "père», aucun autre indice ne montre la présence d'un narrateur "autodiégétique», c'est-à-dire d'un narrateur qui serait aussi le héros de son récit. Au contraire, tout se passe comme si le narrateur de cette scène d'accouchement était «hétérodiégétique ", c'est-à-dire absent de l'histoire qu'il raconte (Genette, p. 253). Le bébé est en effet systématiquement désigné à la troisième personne grammaticale. Les parents "l'attendent de sexe féminin", la sage-femme nettoie «la créature», le père voit «le fils mâle inespérée ${ }^{6}$ » (p. 87). L'emploi de cette troisième personne grammaticale brise le pacte autobiographique. Tout d'abord parce que l'identité entre le personnage et le narrateur n'est pas respectée. Ensuite parce que cela signifie aussi que le narrateur, qui sait tout de la scène qu'il raconte, ne peut pas être le même que l'auteur, qui, lui, ne fait qu'imaginer ce que le lecteur découvre. En d'autres termes, le narrateur fait comme si tout lui était connu de l'histoire qu'il raconte, ce qui ne peut pas être le cas puisqu'il s'agit d'un récit de naissance! De fait, ce n'est que dans la dernière phrase que Cellini s'approprie son prénom et du même coup sa naissance et jusqu'à toute sa personnalité d'homme dans le présent de la narration : "ce prénom me donna le saint Baptême, et je vis ainsi avec la grâce de Dieu ${ }^{47}$ » (p. 87). Ce faisant, il remet le récit sur les rails de l'autobiographie, comme le suggère l'emploi du présent de l'indicatif («je vis») qui fait référence à l'énonciation, c'est-à-dire à la situation du narrateur tel qu'il est au moment où il raconte sa vie.

On peut dès lors s'interroger sur les effets produits par ces ruptures répétées du pacte autobiographique.

Nous avons déjà relevé que le récit de la naissance de Benvenuto ne correspond pas toujours - en apparence - aux critères de l'autobiographie. Un détail à première vue insignifiant peut nous permettre de progresser dans l'analyse. Tous les documents historiques attestent que l'artiste de la Renaissance Benvenuto Cellini est né le 3 novembre I500. Pourtant, dans la Vita, la date de naissance du personnage de Benvenuto est fixée dans la nuit du $\mathrm{I}^{\text {er }}$ au 2 novembre 1500 (p. 87). Or, la façon dont Cellini indique cette date est particulièrement significative. Il ne donne pas d'indication chiffrée, comme on pourrait s'y attendre, mais insiste sur la fête religieuse qui a immédiatement précédé la naissance de Benvenuto : "une nuit de tous les Saints, alors que le jour de la Toussaint était terminé ${ }^{48}$ »; l'importance de cette fête est d'ailleurs soulignée par l'emploi inattendu de

46. "l'aspettavano femmina", "la creatura», "lo inaspettato figliuolo mastio».

47. "tal nome mi diede il santo Battesimo, e così mi vo vivendo con la grazia di Dio ".

48. "una notte di tutti e' Santi, finito il dì d'Ognisanti ". 
l'article indéfini «une» (comme on dirait «une nuit de Noël»), qui donne un aspect légendaire à cette naissance, comme si elle était inscrite hors du temps. L'indication de l'année ne parvient d'ailleurs pas à estomper complètement cette dimension mythique, dans la mesure où elle correspond à l'entrée dans un nouveau siècle, qui draine toujours des attentes millénaristes ${ }^{49}$. À cet égard, il est révélateur que Cellini mette en relief, à l'aide d'un adverbe, la particularité de cette année : "en 1500 précisément ${ }^{\circ \circ}$ " (p. 87).

Pour en revenir au jour précis de la naissance de Benvenuto, la Toussaint est le jour où l'Église catholique honore l'ensemble des saints qu'elle a reconnus, c'est-à-dire l'ensemble de ceux qu'elle reconnaît dignes d'un culte du fait de leur vie exemplaire et de leur proximité avec le divin. L'avancement de vingt-quatre heures de sa date de naissance place donc Benvenuto en communion avec tous les saints. De plus, le 2 novembre est le jour où l'Église honore ses défunts en célébrant des messes pour la rédemption de leurs péchés. Une simple date de naissance donne alors un sens cosmique et mythique à la vie du personnage. Il semble qu'ici le récit de naissance serve à construire la légende même de la personnalité future et du destin hors du commun du personnage Benvenuto.

C'est d'ailleurs bien ce qu'exprime Cellini lorsqu'il décrit la crise mystique qui touche Benvenuto emprisonné trente-neuf ans plus tard. Cellini raconte en effet que Benvenuto a prié Dieu pour qu'il lui montre le soleil, au moins en songe, dans la nuit du 2 au 3 octobre 1539 :

Je pris cette décision et je fis mes plus importantes prières à Dieu le 2 octobre I539. Le matin suivant, qui fut le 3 octobre je m'étais réveillé presque à la pointe du jour, avant le lever du soleil ${ }^{\text {st. }}$. (p. 387)

Benvenuto a alors la vision d'un jeune homme qui lui montre, dans une pièce, l'ensemble des défunts : "Tous ces hommes que tu vois, ce sont tous ceux qui sont nés puis morts jusqu'à maintenant ${ }^{52}{ }^{\prime}$ (p. 387). Cette vision précède celle du soleil, du Christ en croix, puis de la Vierge (p. 389). L'épisode se conclut par un auto-plaidoyer dans lequel Benvenuto, d'un

49. Voir sur ce point C. Vasoli, «L'attesa della nuova èra in ambienti e gruppi fiorentini del Quattrocento", dans L'attesa dell'età nuova nella spiritualità della fine del Medioevo, Convegni del Centro di Studi sulla spiritualità medievale, III (16-19 octobre 1960), Todi, Presso l'Accademia Tubertina, 1962, p. 370-432.

50. «nel mille cinquecento a punto». (C'est nous qui soulignons.)

5I. "Questa resoluzione e queste mie maggior preci a Dio le feci à dì dua d'ottobre nel mille cinquecento trentanove. Venuto poi la mattina seguente, che fu a' dì tre ottobre detto, io m'ero risentito alla punta del giorno, innanzi il levar del sole."

52. "Quelli tanti uomini che tu vedi, sono tutti quei che insino a qui son nati e poi son morti». 
ton menaçant, prévient ses geôliers qu’ils vont devoir le libérer parce qu’il bénéficie de la grâce de Dieu :

Sachez que je suis certain que le jour de tous les Saints, qui fut celui où je vins au monde en 1500 précisément, le premier jour de novembre, la nuit suivante à quatre heures, ce jour qui viendra, vous serez obligés de me sortir de cette sombre prison; et vous ne pourrez pas faire autrement, parce que je l'ai vu de mes yeux et sur ce trône de Dieu $^{53}$. (p. 390)

Nous voyons que la date indiquée pour sa libération est précisément celle de l'anniversaire de sa naissance. Or, cette date correspond ici à celle qui est attestée par les documents historiques. Si bien qu'au niveau de l'histoire racontée dans la Vita se trouve une incohérence chronologique de vingt quatre heures, puisque Benvenuto naît le 2 novembre 1500 au chapitre I-3 et le 3 novembre I500 au chapitre I-I22. C'est que Cellini s'attache moins à la vérité historique des dates qu'à leur signification symbolique. Aussi modifie-t-il, au début de son texte, la date de naissance de Benvenuto. En changeant cette donnée à la fois fondamentale mais a priori anodine de toute autobiographie, Cellini confère d'emblée une dimension mythique et mystique à Benvenuto qui fait de lui, en somme, un saint parmi la cohorte des saints. La dimension hagiographique qui sera conférée au personnage, symbolisée par une auréole censée ceindre la tête de Benvenuto (p. 40o), confirme cette hypothèse.

Dans cette perspective, ce n'est pas un hasard si les parents de Benvenuto évoquent des figures bibliques, celles d'Abraham et de Sara, à qui Yahvé accorde la bénédiction d'une procréation alors qu'ils ont un âge extrême et que Sara n'avait jamais pu enfanter jusqu'alors ${ }^{54}$. De façon semblable, la naissance de Benvenuto est présentée comme un don de Dieu à deux vieillards qui ont attendu dix-huit ans et subi deux fausses couches avant de pouvoir avoir un garçon. Giovanni Cellini rend en effet grâce à Dieu pour cette naissance qui apparaît dès lors presque miraculeuse, d'autant que tout le monde s'attendait à une fille : «Seigneur, je te remercie de tout mon cœur ${ }^{55}$ " (p. 87). La nourrice qui présente le nouveau-né inattendu à son père évoque en outre l'Ange du Seigneur, qui annonce à Zacharie et Élisabeth la naissance de Jean-Baptiste alors que tous deux sont vieux

53. "Sappiate che io sono certissimo che il dì di tutti e' Santi, quale fu quello che io venni al mondo nel mille cinquecento a punto, il primo dì di novembre, la notte seguente a quattro ore, quel dì che verrà, voi sarete forzati a cavarmi di questo carcere tenebroso; e non potrete far di manco, perché io l'ho visto con gli occhi mia e in quel trono di Dio."

54. Genèse, 17, I5-19.

55. "Signiore, io ti ringrazio con tutto il cuor mio". 
et qu'elle était appelée "la stérile ${ }^{56}$ ", d'autant que la mère de Benvenuto porte le même prénom, Elisabetta.

Pour conclure avec Marziano Guglielminetti, ce récit de naissance montre que Benvenuto «n'est pas le fils d'une famille plus ou moins importante, mais plutôt un homme voulu par Dieu et reconnu tout de suite comme un être d'exception ${ }^{57}$ ".

Les premiers chapitres de la Vita confirment le statut unique de son héros. Pour commencer, le prénom Benvenuto annonce un homme prédestiné à l'art, dans la mesure où il se confond avec l'attribut que le père donne au nouveau-né : "Qu'il soit le bienvenu.» Par antonomase au moment du baptême, ce prénom exprime les sentiments de soulagement, de fierté et de reconnaissance qu'éprouve Giovanni Cellini à la naissance de son enfant. Mais il y a plus. Car cette façon de nommer les personnes en fonction de leurs traits de caractère exploite la tradition qui remonte à la Genèse selon laquelle "nomina sunt consequentia rerum ${ }^{88}$ ". Topique depuis l'Antiquité et l'hagiographie, cette tradition a été reprise par la littérature (voir Vita Nuova, XIII, 4 par exemple) et en particulier par la novellistica. Ainsi, dans le Décaméron, le peintre florentin Giovannozzo di Pierino est appelé Calandrino pour exprimer la bêtise et la crédulité chez quelqu'un qui pense au contraire être très malin ${ }^{59}$.

Or, au XVI ${ }^{\mathrm{e}}$ siècle, ce topos a été utilisé dans les biographies des artistes pour suggérer leur prédestination à l'art ${ }^{60}$. Emblématique à cet égard est la biographie de Michel-Ange établie par Vasari, qui indique que Lodovico Buonarroti a nommé son fils "Michele Agnolo" "parce qu'il voulait déduire que celui-ci était une chose plus céleste et divine que mortelle ${ }^{6 \mathrm{I}}$ ».

Le récit de la naissance de Benvenuto dévoile par conséquent un procédé récurrent dans la Vita. À partir de données réelles, Cellini élabore, en s'appuyant sur des schémas littéraires et/ou mythiques, une nouvelle histoire qui fait de Benvenuto un être exceptionnel. Il sublime la réalité, c'est-à-dire qu'il la transforme pour l'élever à un niveau supérieur

56. Évangile selon saint Luc, I, 36.

57. "non è dunque il figlio d'una famiglia più o meno ragguardevole, bensì un uomo voluto da Dio e riconosciuto subito come soggetto di eccezione" (M. Guglielminetti, Memoria e scrittura. L'autobiografia da Dante a Cellini, Turin, Einaudi, 1977, p. 314).

58. Genèse, 2, 20.

59. "Calandrino" est le diminutif de "calandro ", un oiseau de l'espèce des alouettes qui est considéré comme maladroit et stupide.

60. M. L. Altieri Biagi, «La Vita del Cellini: temi, termini, sintagmi», dans Fra lingua scientifica e lingua letteraria, Pise-Rome-Venise-Vienne, Istituti editoriali e poligrafici internazionali, 1998, p. I29-205, ici p. 133-I34.

6I. "volendo inferire costui essere cosa celeste e divina più che mortale" (G. Vasari, Le Vite de più eccellenti architetti, pittori, et scultori italiani, da Cimabue, insino à tempi nostri, édité par L. Bellosi et A. Rossi, Turin, Einaudi, I986, vol. II, p. 88I). 
et construire un destin, anticipant par là l'une des visées traditionnelles de l'autobiographie moderne, centrée sur l'expérience d'un individu qui cherche à se définir par rapport aux autres.

C'est justement dans cette construction d'un personnage unique que l'exploitation des caractéristiques de la nouvelle se révèle particulièrement efficace. Loin d'éloigner alors le texte de la Vita du projet autobiographique, ces digressions en constitueraient en définitive un élément constitutif.

L'épisode où Benvenuto enfant échappe à la morsure d'un scorpion est particulièrement emblématique. La présence de la famille tout entière, exprimée par l'emploi de la troisième personne du pluriel, non seulement appose le sceau de la vraisemblance sur les événements qui sont racontés, mais donne une vision idéalisée de l'enfance de Benvenuto, qui confine au mythe. Car plus que l'anecdote en elle-même, c'est le sens que lui donne Cellini qui est important. Le narrateur rapporte en effet que Giovanni considéra l'incident «de bon augure» ("per buono aurio»). Cette projection dans le futur fait de cet épisode le mythe fondateur de Benvenuto, ce personnage qui peut serrer de toutes ses forces un animal venimeux sans en subir aucune conséquence fâcheuse. L'invincibilité de Benvenuto est du reste soulignée par l'hyperbole employée pour décrire la bête, qui, raconte Cellini, "était si grande que, lorsque je l'avais dans la main, sa queue dépassait d'un côté et ses deux pinces dépassaient de l'autre ${ }^{62}$ » (p. 88).

D'ailleurs, le scorpion étant le signe de Benvenuto sur l'horoscope, instrument de prévision auquel les hommes de la Renaissance prêtaient la plus grande attention, on peut voir dans cette scène une façon d'annoncer que Benvenuto saura être maitre de son destin. De plus, dans la tradition grecque, le scorpion était l'instrument de la justice vindicative ${ }^{63}$. Or, la Vita est aussi la mise en scène d'un personnage qui se venge de toutes les injustices qu'il estime subir, la première étant le manque de reconnaissance de son talent par le duc Cosme de Médicis, qui a justement déclenché le processus autobiographique. Le scorpion apparaît alors comme l'instrument qui ouvre les portes du monde à l'artiste.

L'ensemble de cette scène porte donc déjà la marque de ce que Françoise Duranton-Mallet appelle le «fantasme d'exception», qu'on retrouve ensuite

62. "era sì grande, che avendolo innella picciola mano, da uno degli lati avanzava fuori la coda, e da l'altro avanzava tutt'a due le bocche».

63. J. Chevalier et A. Gheerbrant, Dictionnaire des symboles, Paris, Robert Laffont, Bouquins, I969, p. 855. 
tout au long de la Vita ${ }^{64}$. De ce point de vue, l'absolution de Benvenuto par le pape Paul III pour l'homicide de Pompeo de' Capitaneis est significative, puisque l'artiste se voit échapper aux lois qui régissent le commun des mortels : "Sachez que les hommes comme Benvenuto, uniques dans leur profession, n'ont pas à être contraints par la loi ${ }^{65} . "$ (p. 265)

C'est que Benvenuto n'est pas seulement un don de Dieu. Il est aussi fils de prophète... Giovanni Cellini prédit ainsi l'élection du cardinal Giovanni de’ Medici sur le trône pontifical grâce à «sa fibre prophétique, ce qui était certainement divin chez lui ${ }^{66}{ }^{\prime}$ (p. 92). Surtout, c'est par des «mots prophétiques» ("parole profetiche», p. 97), que Giovanni prédit les malheurs qui sabattront sur le joueur de fifre Pierino, dans le chapitre I-9 que nous avons évoqué au début de cette analyse. Lépisode pourrait n'être qu'une vérification de la lucidité et de la clairvoyance de Giovanni si Cellini ne lui donnait pas une valeur symbolique très forte et s'il ne l'interprétait pas comme une manifestation de la présence divine chez son père: "que personne ne se moque jamais des présages d'un homme de bien lorsqu'il a été injustement injurié, parce que ce n'est pas lui qui parle, mais il est la voix de Dieu lui-même ${ }^{67}$ » (p. 99). Si le ton sentencieux et menaçant résonne comme un avertissement lancé à quiconque pourrait s'en prendre à la famille de Giovanni, autrement dit à quiconque oserait s'en prendre à Benvenuto, cette phrase finale clôt l'épisode sur le modèle des nouvelles traditionnelles.

Le dénouement de l'épisode a en effet une valeur moralisatrice. Pierino a enfreint les règles des rapports humains, d'abord en ne respectant pas les ambitions qu' un père (Giovanni) nourrissait pour son fils (Benvenuto), ensuite en faisant preuve d'ingratitude vis-à-vis de son maître, enfin en se moquant des avertissements de ce dernier. Pierino mérite alors d'être sanctionné pour son arrogance. Aussi est-il doublement puni : par la mort pour lui-même, mais aussi par la pauvreté qui accable son fils. On peut d'ailleurs remarquer que Pierino est puni par là même où il accablait injustement Giovanni : alors qu'il accusait le père de Benvenuto de dilapider sa fortune et de ne rien laisser à ses enfants, c'est lui qui abandonne son fils sans ressources et le contraint à demander la charité à Benvenuto.

64. F. Duranton-Mallet, "Propositions pour une lecture analytique de la Vita de Benvenuto Cellini», Revue des Études italiennes, 1983, XXIX, p. 223-23I, ici p. 225.

65. "Sappiate che gli uomini come Benvenuto, unici nella lor professione, non hanno da essere ubrigati alla legge."

66. "vena profetica, che questo certo era divino in lui».

67. «nessuno non si faccia mai beffe dei pronotischi di uno uomo da bene, avendolo ingiustamente ingiuriato, perché non è lui quel che parla, anzi è la voce de Idio istessa». 
Récit bref, autonome et auto-suffisant, centré sur un seul événement qui se termine par une conclusion édifiante, le chapitre I-9 a toutes les caractéristiques d'une nouvelle, que Cellini insère dans la Vita pour camper le personnage de Giovanni. Tout se passe en effet comme si le statut particulier de Benvenuto, qui est à la fois l'élu de Dieu et le fils d'un prophète, exigeait un texte extra-ordinaire, dont la trajectoire ne suivrait pas, de façon rectiligne, le fil du récit initial.

La façon dont Pierino est puni évoque en outre un trait récurrent dans la novellistica: il s'agit de la beffa, qui consiste, dans une relation de supérieur à inférieur, à donner une leçon au sot et/ou à l'arrogant qui a commis une erreur d'ordre moral ou intellectuel ${ }^{68}$. Surtout, la mort de Pierino permet à Benvenuto d'exercer la charité avec une générosité et une noblesse destinées à susciter l'admiration du lecteur («je lui fis l'aumône, car c'est ma nature de faire l'aumône ${ }^{69}$ ", p. 99).

Cellini exploite le même schéma dans le chapitre I-30. Même si les personnages sont réunis autour de Benvenuto pour célébrer la fin de la peste et non pour l'oublier, comme le font les jeunes gens du Décaméron ou des Cene de Grazzini, le défi initial lancé par les convives renvoie aux situations plus ou moins scabreuses des nouvelles traditionnelles: il s'agit de participer à un souper accompagné de sa courtisane favorite, exigence qui confere immédiatement une dimension érotique à l'épisode. Le récit est ensuite entièrement placé sous le signe du comique, qui naît de la transgression des normes sociales, et plus précisément des normes sexuelles et religieuses : le travestissement de Diego en femme d'une part, et son imitation de la bénédiction papale d'autre part, enchantent les invités. Mais c'est surtout le dénouement de l'histoire qui rattache l'épisode à la nouvelle. Diego, excédé par la bêtise des femmes qui jacassent autour de lui, se tortille, feignant d'avoir l'utérus douloureux. Prises de pitié, ses voisines de table cherchent à le soulager en le touchant et s'aperçoivent alors de la supercherie... à leur grande surprise et colère, mais aussi à la grande joie des invités de sexe masculin! La beffa est destinée ici à sanctionner la bêtise des femmes jugées idiotes. Mais elle permet en même temps à Cellini de mettre en valeur le héros de la Vita.

La transformation de Diego en Pomona exalte en effet le sens esthétique de Benvenuto. L'admiration que les convives éprouvent devant la beauté de Diego-Pomona est révélatrice du talent de l'artiste, qui a paré

68. Sur la beffa, voir Formes et significations de la "beffa" dans la littérature italienne de la Renaissance, édité par A. Rochon, Paris, Université de la Sorbonne Nouvelle, 1972, vol. I et 1975, vol. 4.

69. "Io gnene diedi, sì per esser mia natura il far delle elemosine». 
son ami de bijoux comme il aurait façonné une statue (p. I49). De plus, la tromperie est entièrement valorisée au bénéfice du personnage Benvenuto, puisque le tour qu'il a joué lui vaut d'être acclamé à la fin du souper pour son habileté et son imagination.

À travers les aventures amoureuses de Benvenuto avec Angelica, c'est l'esprit de répartie de Benvenuto que Cellini met en valeur dans le cadre d'une «nouvelle qui semble tirée du Decameron ${ }^{70}$ ". Même si Cellini puise de temps à autre au roman sentimental de la tradition grecque ${ }^{71}$ ainsi qu'aux romans chevaleresques ${ }^{72}$, les chapitres 63 à 70 de la première partie empruntent en effet des traits à la novellistica. On peut songer par exemple à l'érotisme effréné auquel s'adonne Benvenuto, seul remède à ses tourments d'âme éprise, mais contrariée par le départ de sa belle : "[...] en ce temps-là j'avais goûté à tous les plaisirs qu'on peut imaginer, et $\mathrm{j}$ 'avais pris un autre amour, uniquement pour éteindre celui-ci ${ }^{73}$.» $\mathrm{La}$ scène scatologique qui se déroule au Colisée ${ }^{74}$ renvoie elle aussi à une thématique récurrente des nouvelles. Surtout, le dénouement s'inspire des chutes caractéristiques de la novellistica. Alors que Benvenuto a déjà fait montre d'une grande générosité envers Angelica et sa mère, celle-ci verrait bien que les largesses de l'amant soient encore plus abondantes. Cellini relate alors un mini-dialogue qui fonctionne sur l'efficacité du langage. Tout comme les personnages de nouvelles qui se défendent par l'humour, Benvenuto donne une leçon à la mère d'Angelica grâce au motto, le mot d'esprit. L'échange de répliques vaut la peine d'être cité :

Je me tournai avec grâce vers elle et je lui dis : "Ma chère Beatrice, ce que je t’ai offert te suffit-il?” Elle dit que non. Alors je lui dis que ce qui ne lui suffisait pas, à elle,

70. E. Carrara, «Manierismo letterario in Benvenuto Cellini», Studi romanzi, ${ }^{\circ}$ 19, 1928, p. 171-200, ici p. 189 .

7I. La rencontre, la séparation et les épreuves subies avant les retrouvailles peuvent évoquer les aventures de Leucippé et Clitophon racontées par Achille Tatius d'Alexandrie, texte qui avait d'ailleurs également inspiré Annibal Caro.

72. Portant le même prénom que la bien-aimée de Roland, Angelica est aussi source de folie ou de mort pour Benvenuto ("je fus sur le point ou de devenir fou ou de mourir", « io stetti in procinto o d'inpazzare o di morire", p. 239), tout comme elle rend Roland fou lorsqu'elle s'enfuit avec Medoro. Par ailleurs les éléments surnaturels qui animent la séance de nécromancie au Colisée (l'apparition de légions diaboliques, le cercle magique, le parfum, le feu, les visions d'un enfant vierge, la prédiction concernant la rencontre entre Benvenuto et Angelica), évoquent le merveilleux des romans chevaleresques, les légions diaboliques pouvant par exemple s'apparenter à la forêt, lieu topique représentant les obstacles à surmonter, et le nécromancien incarnant l'actant adjuvant qui favorise la quête du héros, à savoir son amour pour Angelica.

73. «[...] in questo tempo io avevo atteso a tutti i piaceri che inmaginar si possa, e avevo preso altro amore, solo per istigner quello.» (p. 240)

74. "Cet Agniolo, au moment où il voulut bouger, lâcha un concert de pets avec une telle quantité de merde, qu'elle fut plus efficace que l'assa-fœetida» ("Il ditto Agniolo, in quello che lui si volse muovere, fece una strombazzata di coreggie con tanta abundanzia di merda, la qual potette più che la zaffetica»), p. 243. 
me suffirait, à moi; et après avoir embrassé mon Angelica, nous nous séparâmes, elle éplorée, moi hilare, et je m’en retournai tout de suite à Rome ${ }^{75}$. (p. 254)

L'ironie est fondée ici d'une part, sur la reprise de termes identiques (le verbe "suffire») dans un parallélisme inversé au niveau du sens et d'autre part, sur le contraste des sentiments des deux anciens amants. Comme dans les nouvelles, le motto permet à Benvenuto de donner une leçon à un personnage qui cherchait à lui nuire : il a préservé ses richesses tout en rappelant les règles de la politesse.

Mais il y a plus. Les artistes incarnent souvent dans la novellistica cette ingéniosité de l'esprit qui est le moteur de la beffa ou du motto. Des peintres Bruno et Buffalmacco qui jouent des tours à Calandrino ou au médecin Simone au cours de la huitième journée du Décaméron (nouvelles 3, 6 et 9), au duo constitué par le peintre Scheggia et le sculpteur et architecte Pilucca dans les Cene (I-3; II-4 et II-6), en passant par le peintre Bonamico dans le Trecentonovelle (nouvelles I69, I9I et 192 par exemple), les artistes sont les "beffatori par excellence», comme le fait remarquer Bruscagli $^{76}$. Si bien qu'exploiter dans la Vita ces schémas récurrents de la novellistica peut être une façon de donner à Benvenuto un statut social digne des autres artistes.

Le silence de Vasari dans sa première édition des Vite $e^{77}$ sur la carrière de Benvenuto Cellini ainsi que le manque de reconnaissance du duc Cosme de Médicis après la réalisation du Persée ont en effet poussé notre artiste à prouver aux autres sa valeur. "Après qu'on m'a empêché de faire, je me suis ainsi mis à dire ${ }^{78}$ ", écrit-il dans son traité sur l'orfevrerie à propos de la Vita.

Dans cette perspective, il est significatif que Benvenuto se libère du pouvoir aliénant de ses commanditaires à l'aide de mots d'esprit. C'est par la parole qu'il peut revendiquer sa liberté de créer et le statut social qui en découle, comme lorsqu'il défend son indépendance d'artiste vis-à-vis du cardinal de Ferrare. L'émissaire de ce dernier le pressant de se mettre en route rapidement pour répondre à l'appel du roi de France, il s'ensuit cet

75. "Alla quale io piacevolmente mi volsi e le dissi: "Beatrice mia cara, bastat'egli quello che io t'ho offerto?" Lei disse che no. Allora io dissi che quel che non bastava a lei basterebbe a me; e baciato la mia Angelica, lei con lacrime e io con riso ci spiccammo, e me ne tornai a Roma subito."

76. R. Bruscagli, «La novella e il romanzo", dans Storia della letteratura italiana, édité par E. Malato, Rome, Salerno Editrice, 1996, vol. IV : Il primo Cinquecento, p. 835-906, ici p. 858. Sur la présence des artistes dans les nouvelles, voir J. Von Schlosser, La littérature artistique. Manuel des sources de l'histoire de l'art moderne, Paris, Flammarion, 1996, p. 90, et M. L. Altieri Biagi, art. cité, p. I57.

77. Sur ce point, on consultera Giulia Dell'Aquila, "'Con artifizio maravigliosissimo'. Benvenuto Cellini nelle biografie d'artisti tra Cinquecento e Settecento ", Letteratura e arte, no I, 2003, p. 227-244.

78. "da poi che m’è impedito il fare, così io mi son messo a dire" (B. Cellini, Dell'Oreficeria, op. cit., p. 718). 
échange fondé sur un jeu de mots qui s'articule autour du temps nécessaire, pour se déplacer d'une part, et pour créer d'autre part :

Il me dit de m’organiser rapidement, pour que j’aille très rapidement. Je lui répondis que mon art ne se faisait pas très rapidement, et que si j'avais à me déplacer, je voulais le faire avec des étapes agréables et emmener avec moi Ascanio et Pagolo, mes ouvriers, que j'avais fait partir de Rome; et qu'en outre je voulais un serviteur à cheval avec nous, à mon service, et suffisamment d'argent pour mon voyage. Ce vieil infirme me répondit par des mots remplis de superbe que c'étaient uniquement les enfants du Duc qui se déplaçaient de cette façon-là. Je lui répondis aussitôt que les enfants de mon art allaient de la façon que j'avais dite; et qu'étant donné que je n’avais jamais été fils de duc je ne savais pas comment ceux-là se déplaçaient ${ }^{79}$. (p. 424-425)

Le chapitre I-6o, dans lequel Benvenuto refuse de faire le calice du pape Clément VII fonctionne sur le même principe. L'artiste exige tout d'abord du pape qu'il respecte ses engagements. En tant que commanditaire, il doit fournir l'or à l'artiste, parce que, dit un Benvenuto ironique et provocateur qui joue sur l'efficacité d'une comparaison triviale empruntée à la vie quotidienne : "dites-moi donc comment on peut faire le pain sans farine? de la même façon cette œuvre ne sera jamais finie sans $\mathrm{or}^{80}$ » (p. 23I). Benvenuto revendique ensuite la propriété de ses créations : «l'œuvre est à moi, et j'en ferai ce qu'il me plaît ${ }^{81}$ » (p. 232). Lorsque les serviteurs du pape viennent exiger le calice, l'artiste réplique du tac au tac, jouant sur les adjectifs et pronoms possessifs : «si je donnais l'œuvre à sa Sainteté, je donnerais mon œuvre et non la sienne ${ }^{82}$ " (p. 233). Or, c'est «joyeusement» («lietissimamente») que Benvenuto s'adresse à eux, comme si Cellini voulait indiquer que le langage est un jeu. Enfin, Benvenuto exige la reconnaissance de son talent, qui ne saurait être mesuré comme une vulgaire marchandise, "parce que [le calice] n'est ni une maison, ni un bijou ${ }^{{ }^{3}}$ » (p. 235). Benvenuto utilise donc la parole pour défendre son talent et son statut social, à la façon d'un Giotto qui ridiculise un artisan dans la nouvelle 63 du Trecentonovelle. L'artiste mis en scène par Sacchetti, irrité par la prétention et l'outrecuidance d'un artisan parvenu qui exige

79. «[...] mi disse che io mi mettessi in ordine presto, per correre in poste. Al quale io dissi che l'arte mia non si faceva in poste, e che se io vi avevo da 'ndare, volevo andarvi a piacevol giornate e menar meco Ascanio e Pagolo, mia lavoranti, i quali avevo levati di Roma; e di più volevo un servitore con esso noi a cavallo, per mio servizio, e tanti danari che bastassino a condurmivi. Questo vecchio infermo con superbissime parole mi rispose che in quel modo che io dicevo, e non altrimenti, andavano i figliuoli del Duca. A lui subito risposi che i figliuoli de l'arte mia andavano in quel modo che io avevo detto; e per non essere stato mai figliuol di duca quelli non sapevo come s'andassino."

80. «insegnatemi un poco come sanza farina si può fare il pane? così sanza oro mai si finirà quell'opera».

8I. "l'opera è mia, e ne farò quanto m'è di piacere».

82. "se io dessi l'opera a sua Santità, io darei l'opera mia e non la sua».

83. "perché la non è né una casa, né una gioia". 
de lui qu'il peigne ses armoiries («arme» en italien) sur un pavois, peint des armes militaires et se justifie en jouant sur la double signification du mot «arme».

En ce qui concerne la Vita, les épisodes qui vont dans le sens d'une valorisation de Benvenuto sont innombrables. Le mettre en contact avec frère Pallavicini, qui, parce qu'il «s'abandonnait à toutes les formes de vices $^{84}$ » (p. 346), évoque aussi bien les religieux corrompus que Tebaldo décrit dans la nouvelle III, 7 du Décaméron que la figure du diable tentateur, récurrente dans la novellistica, permet par exemple de montrer que le héros de la Vita tient sa parole en dépit des tentations. Mais il est surtout important, au terme de cette étude, de souligner que c'est la reprise de schémas littéraires préexistants, et en particulier celui de la nouvelle, qui permet à Cellini de valoriser son personnage.

Si une partie de notre analyse pourrait dès lors être appliquée à la présence dans la Vita d'autres genres littéraires comme le théâtre, l'épopée ou le roman picaresque, la nouvelle a toutefois ceci de particulier que, lorsqu' elle n'est pas unique ("spicciolata» en italien) et qu'elle s'insère dans un recueil, elle dépend d'une nouvelle cadre ${ }^{85}$ - la cornice dans la tradition italienne ${ }^{86}$. Or, même si certains extraits de la Vita, marqués par l'unité spatiale, temporelle et événementielle, forment une entité autosuffisante, chacune des nouvelles que nous avons évoquée apparaît en réalité comme une composante de la mosaïque que Cellini élabore pour décrire Benvenuto. De sorte que les nouvelles sont liés dans la Vita par enchâssement et non par enchaînement : elles ne sont pas juxtaposées les unes aux autres et coordonnées par un récit-cadre commun, mais chacune d'elle est incluse dans le récit que Cellini fait de sa vie. Ainsi, chacune des nouvelles de la Vita procède de l'ensemble : parce qu'on est dans le cadre d'une autobiographie, chacune est subordonnée au personnage même de Benvenuto, omniprésent et hyperbolique, qui assure la cohésion, si ce n'est la continuité, entre les épisodes.

C'est que dans le cadre des nouvelles énoncées à la première personne du singulier, «le narrateur prend le pas sur l'histoire, la nouvelle cesse d'être récits d'événements au profit d'une relation révélatrice d'une personne»

84. "s'accomodava a tutte le sorte de' vizii».

85. V. Chklovski, "La construction de la nouvelle et du roman», dans Théorie de la littérature, édité par T. Todorov, Paris, Seuil, 1965, p. 170-196, ici p. 170.

86. Il est intéressant pour notre propos de noter que Bruscagli fait de la cornice la raison pour laquelle les nouvelles pénètrent facilement dans les autres genres littéraires (voir Bruscagli, art. cité, p. 839). Sur la cornice, voir M. Ciccuto, "Introduzione», Novelle italiane. Il Cinquecento, Milan, Garzanti, 1982, p. Xv-xviII et surtout M. Guglielminetti, La cornice e il furto. Studi sulla novella del 'soo, Bologne, Zanichelli, I984. 
(Grojnowski, p. I28-I29). Nous avons vu en effet comment les nouvelles présentent une facette, toujours flatteuse, du protagoniste Benvenuto.

Mais d'une certaine façon, et pour en revenir à la citation initiale de Paul Bourget, on pourrait dire que la Vita de Cellini se trouve entre la nouvelle et le roman. C'est d'ailleurs ce qui en fait sa modernité. En effet, selon Chklovski, le Décaméron «est encore très différent du roman européen des XVIII ${ }^{e}$ et $\mathrm{XIX}^{\mathrm{e}}$ siècles parce que les mêmes personnages ne lient pas les épisodes particuliers» (Chklovski, p. 190). Au contraire, nous avons vu que la Vita montre des épisodes liés les uns aux autres par un élément constant : le personnage de Benvenuto ${ }^{87}$. Toutefois, la Vita partage avec le recueil de Boccace le fait que "l'attention se concentre sur l'action" (Chklovski, p. 190). C'est que Benvenuto se définit davantage par ses actes que par l'introspection. Les regrets qu'il exprime à la lumière du présent vis-à-vis d'une décision prise dans le passé, comme de ne pas être parti en France par exemple, font ainsi figure d'exception. Rares sont en effet les moments où le «je actuel» (l'auteur-narrateur) s'attarde sur les moments $\mathrm{du}$ «moi révolu», pour employer les catégories définies par Starobinski ${ }^{88}$.

Pour conclure, "la Vita de l'artiste, bien que rythmée par les événements professionnels, est une sorte de Décaméron à la première personne, dont l'unité n'est plus assurée par une "cornice", mais se réalise de l'intérieur par l'omniprésence du protagoniste ${ }^{89}{ }^{»}$. Mais cette contamination de l'autobiographie par la nouvelle soulève une interrogation : comment le pacte autobiographique peut-il être respecté quand dans le même temps le personnage dont la vie est racontée est une élaboration qui relève, sinon de la fiction, du moins de la sublimation?

La réponse ne se trouve ni du côté du narrateur Cellini (qui noue le pacte autobiographique avec le lecteur) ni du côté du personnage Benvenuto (qui est l'incarnation d'un héros exceptionnel) mais du côté de l'auteur Benvenuto Cellini (qui préexiste au narrateur). Car cet artiste né à Florence le 3 novembre I500 veut être «le premier homme du monde de $[\mathrm{sa}]$ profession $^{90} »($ p. 516). De sorte que dans la Vita l'élaboration du

87. L’omniprésence de ce personnage éclaire d'ailleurs la position de Nino Borsellino, pour qui l'œuvre de Cellini marque justement le passage de la biographie au roman (N. Borsellino, "Cellini scrittore», dans Benvenuto Cellini artista e scrittore (Rome-Florence, 8-9 février 197I), Rome, Accademia nazionale dei Lincei, I972, p. I7-3I, ici p. 26).

88. J. Starobinski, «Le style de l'autobiographie», Poétique, n 3, I970, p. 257-265.

89. "La Vita dell'artista, pur scandita dagli eventi professionali, è una specie di Decameron in prima persona, la cui unità non si affida più a una 'cornice', ma si realizza dall'interno con l'onnipresenza del protagonista" (Altieri Biagi, art. cité, p. I60).

90. «il primo uomo del mondo della [sua] professione». 
personnage autour de la sublimation de la réalité fait partie, tout comme l'établissement du pacte autobiographique avec le lecteur, d'une même stratégie : il s'agit pour Benvenuto Cellini de revendiquer un statut d'artiste dans la société florentine de la fin $\mathrm{du} \mathrm{XVI}^{\mathrm{e}}$ siècle et de prendre une revanche sur ses adversaires et les "étoiles perverses» ("perverse stelle») qu'il a mis tant d'énergie à combattre.

Ainsi, loin du simple compte rendu de sa propre existence, Cellini fait de la Vita une représentation de lui-même. La Vita n'est pas pour autant une œuvre de fiction, puisque l'auteur est aussi le personnage. Et quand bien même certains événements de sa vie seraient modifiés, supprimés ou inventés, ces transformations découlent toutes du regard que Cellini veut porter sur lui-même. Dès lors «nous ne sommes plus dans le domaine de la vérité (de l'histoire véridique), nous sommes désormais dans celui de l'authenticité (du discours authentique) ${ }^{91}{ }^{\text {}}$, fondée sur la vérité de Benvenuto Cellini au moment où il se raconte.

Dans cette perspective, l'exploitation de la tradition de la nouvelle est destinée à construire cette auto-représentation valorisante de soi-même, en suscitant l'admiration des lecteurs. Chaque épisode, autonome et autosuffisant, permet de brosser un portrait héroïque de l'artiste. Rassemblés, ces épisodes constituent ce qu'on pourrait appeler le «roman personnel» de Benvenuto Cellini, qui livre à la postérité une image idéalisée de luimême. À travers les nouvelles qui contribuent à façonner le portrait de Benvenuto, Cellini fait connaître moins sa vie telle qu'elle a été que sa vie telle qu'il veut la présenter aux lecteurs.

À la fin de la Vita, le présent de l'auteur Benvenuto Cellini finit d'ailleurs par envahir la scène autobiographique. Comme le souligne Corinne Lucas, "le temps de l'énoncé et celui de l'énonciation finissent par se recouvrir et se superposer ${ }^{92} »$. Ainsi, lorsque Cellini fait allusion à la composition d'Apollon qui se trouve "encore aujourd'hui» dans son atelier, il s'agit autant d'une reconstruction a posteriori du passé que de la description contemporaine du présent : "j'en tirai ce que je pus, à savoir Apollon et Hyacinthe, dont on peut encore voir l'imperfection dans ma boutique ${ }^{93}$ " (p. 559). L'emploi du présent de narration "si vede» souligne cette simultanéité entre les deux facettes de l'homme Benvenuto Cellini, celui d'hier qui est décrit et celui d'aujourd'hui qui se raconte. Mais au fil

91. J. Starobinski, Jean-Jacques Rousseau. La transparence et l'obstacle, Paris, TEL Gallimard, I97I, p. 237.

92. C. Lucas, "Remarques sur le temps dans la Vita de Benvenuto Cellini», Chroniques italiennes, n I, I994, p. 47-73, ici p. 7I.

93. "ne cavai quel io potetti, che è l'Apollo e Iacinto, che ancora si vede inprefetto in bottega mia». 
des pages non seulement la distance temporelle entre l'auteur-narrateur et le personnage s'atténue, mais l'auteur-narrateur tend même à dépasser le personnage. Car lorsque Benvenuto accepte un contrat en viager, il se projette dans le futur, un futur qui n'est justement pas celui du personnage Benvenuto, mais bien celui de l'auteur Benvenuto Cellini évoqué par le narrateur Cellini : «je m'efforcerai de vivre le plus longtemps possible ${ }^{94}$ » (p. 643).

Mais si le temps du récit rejoint le temps de l'écriture, s'il n'y a plus de distance entre l'énoncé et l'énonciation, c'est que la transfiguration du personnage en héros s'est définitivement accomplie et le silence s'impose. De fait, Cellini interrompt brutalement la Vita peu après cette observation. 
